\title{
FUTURE TRENDS IN FIBER OPTICS COMMUNICATION
}

\author{
Ankur tiwari \\ B.tech $4^{\text {th }}$ Yr \\ AIET, JAIPUR
}

\author{
Raj Kumar \\ B.tech $4^{\text {th }} \mathrm{Yr}$ \\ Arya Institute of Engineering and Technology
}

\author{
Abhishek Saxena \\ Asst. prof. \\ Arya Institute of Engineering and Technology
}

\begin{abstract}
Fiber optic systems area unit vital broadband networks. Wide information measure signal Un transmission with low delay could be a key demand in oift $c$ day applications. Optical fibers give huge and unexceeded

of fiber optic communication systems together with their key technologies, and conjointly discusses their technological trend towards future generation.
\end{abstract} transmission information measure with negligible latency, Several new classes of optical communication networks are and area unit currently the transmission medium of presently emerging [2]. For example, Code Division Multiple alternative for long distance and high rate transmission in Access networks using optical signal processing techniques telecommunication networks. This paper offers an outline have recently being introduced [3].

Keywords- Bandwidth, Broadband, Fiber optics, Latency, Telecommunication.

\section{INTRODUCTION}

The major driving force behind the widespread use of fiber optics communication is the high and rapidly increasing consumer and commercial demand for more telecommunication capacity and internet services, with fiber optic technology capable of providing the required information capacity (larger than both wireless connections and copper cable). Advances in technology have enabled more data to be conveyed through a single optical fiber over long distances. The transmission capacity in optical communication networks are significantly improved using wavelength division multiplexing [1].

A desirable feature for future optical networks is the ability to process information entirely in the optical domain for the purpose of amplification, multiplexing, demultiplexing, switching, filtering, and correlation, since optical signal processing is more efficient than electrical signal processing.

Francis Idachaba is a senior lecturer in the Department of Electrical and Information Engineering, Covenant University, Nigeria (e-mail: francis.idachaba@ covenantuniversity.edu.ng).

Dike U. Ike is a post-graduate student in the Department of Electrical and Information Engineering, Covenant University, Nigeria (e-mail: engineerdikeike@yahoo.com).
Despite the associated benefits of utilizing optical fiber for communication (such as its high reliability over long distances, low attenuation, low interference, high security, very high information capacity, longer life span and ease of maintenance), research is still ongoing to further improve on the present fiber optics communication system, and also to solve some of the challenges facing it. Future optical communication systems are envisioned to be more robust than the present system. This paper is organized as follows. Section II describes the basic principles of fiber optics communication. Section III looks at the history and evolution of fiber optics communication while section IV presents some envisioned future trends in fiber optics communication. In section $\mathrm{V}$, we draw the conclusion for the paper.

\section{BASIC PRINCIPLES OF FIBER OPTIC} COMMUNICATION

Fiber optic communication is a communication technology that uses light pulses to transfer information from one point to another through an optical fiber. The information transmitted is essentially digital information generated by telephone systems, cable television companies, and computer systems. An optical fiber is a dielectric cylindrical waveguide made from low-loss materials, usually silicon dioxide. The core of the waveguide has a refractive index a little higher than that of the outer medium (cladding), so that light pulses is guided along the axis of the fiber by total internal reflection [4]. Fiber optic communication systems consists of an optical transmitter to convert an electrical signal to an optical signal for transmission through the optical fiber, a cable containing several bundles of optical fibers, optical amplifiers to boost the power of the optical signal, and an optical receiver to reconvert the received optical signal back to the original transmitted electrical signal. Figure 1 gives a simplified description of a basic fiber optic 
communication system.

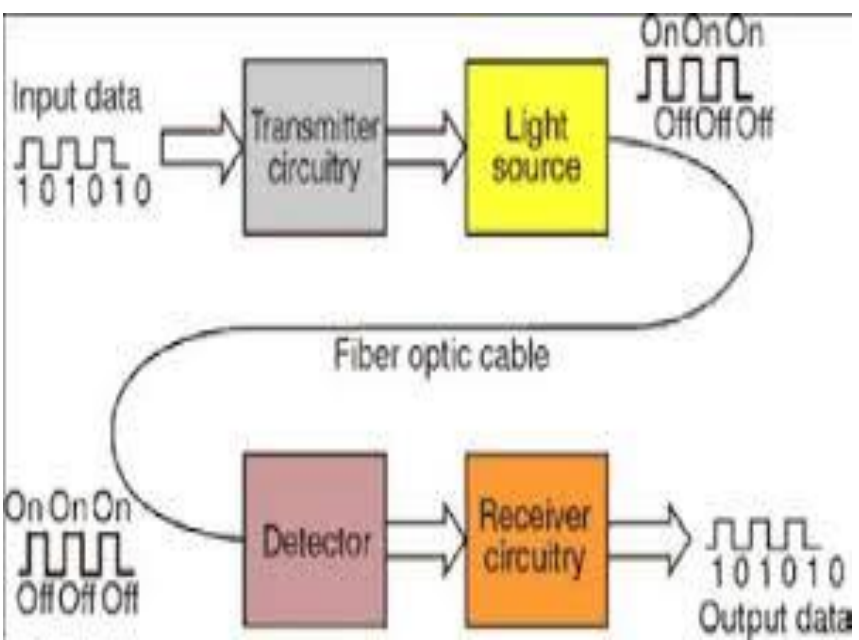

Fig.1. Basic fiber optic communication system [5]

Optical fibers fall into two major categories, namely: step index optical fiber, which include single mode optical fiber and multimode optical fiber, and graded index optical fiber. Single mode step index optical fiber has a core diameter less than 10 micrometers and only allows one light path. Multimode step index optical fiber has a core diameter greater than or equal to 50 micrometers and allows several light paths, this leads to modal dispersion. Graded index optical fibers have their core refractive index gradually decrease farther from the centre of the core, this increased refraction at the core centre slows the speed of some light rays, thereby allowing all the light rays to reach the receiver at almost the same time, thereby reducing dispersion. Figure 2 gives a description of the various optical fiber modes.
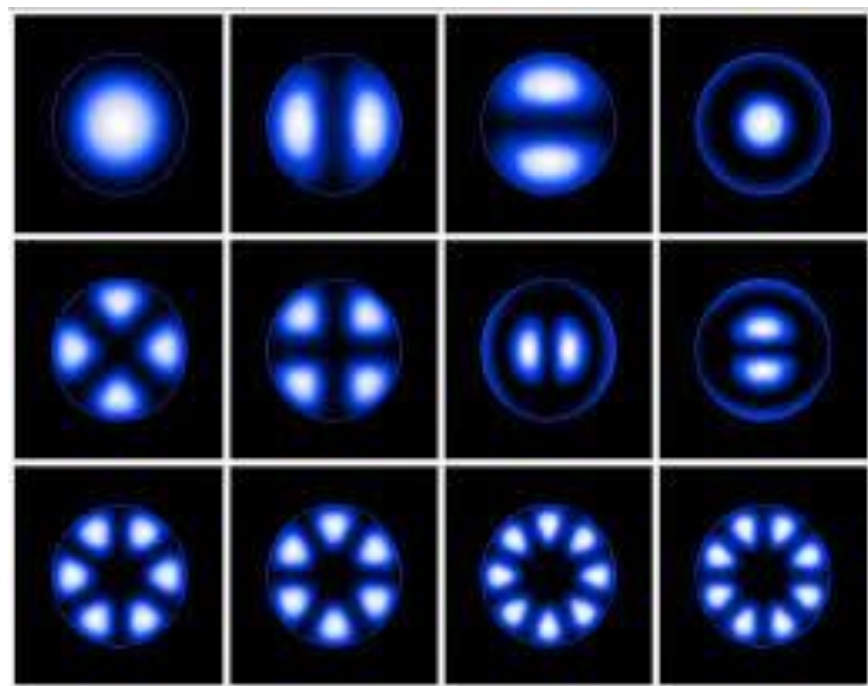

Fig.2. Optical Fiber Modes [6]

\section{EVOLUTION OF FIBER OPTICS \\ COMMUNICATION}

Optical fiber was first developed in 1970 by Corning Glass Works. At the same time, GaAs semiconductor lasers were also developed for transmitting light through the fiber optic cables. The first generation fiber optic system was developed in 1975, it used GaAs semiconductor lasers, operated at a wavelength of $0.8 \mu \mathrm{m}$, and bit rate of $45 \mathrm{Megabits} / \mathrm{sec}$ nd with $10 \mathrm{Km}$ repeater spacing.

In the early 1980's, the second generation of fiber optic communication was developed, it used InGaAsP semiconductor lasers and operated at a wavelength of 1.3 $\mu \mathrm{m}$. By 1987, these fiber optic systems were operating at bit rates of up to 1.7 Gigabits/second on single mode fiber with $50 \mathrm{Km}$ repeater spacing.

The third generation of fiber optic communication operating at a wavelength of $1.55 \mu \mathrm{m}$ was developed in 1990. These systems were operating at a bit rate of up to 2.5 Gigabits/second on a single longitudinal mode fiber with $100 \mathrm{Km}$ repeater spacing.

The fourth generation of fiber optic systems made use of optical amplifiers as a replacement for repeaters, and utilized wavelength division multiplexing (WDM) to increase data rates. By 1996, transmission of over $11,300 \mathrm{Km}$ at a data rate of $5 \mathrm{Gigabits} / \mathrm{sec}$ ond had been demonstrated using submarine cables [7].

The fifth generation fiber optic communication systems use the Dense Wave Division Multiplexing (DWDM) to further increase data rates. Also, the concept of optical solitons, which are pulses that can preserve their shape by counteracting the negative effects of dispersion, is also being explored. Figure 3 shows the evolution of fiber optic communication.

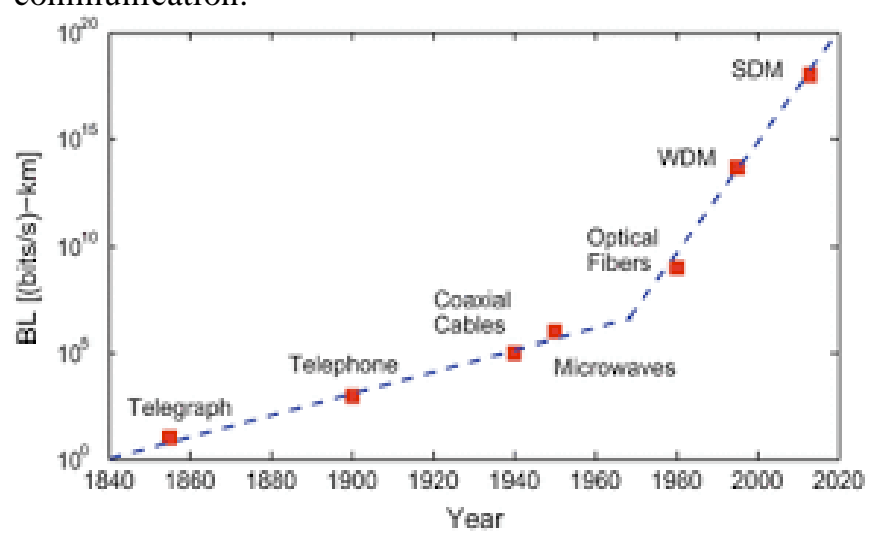

Fig.3. Generations of Fiber Optics Communication [8]

\section{FUTURE TRENDS IN FIBER OPTICS COMMUNICATION}

Fiber optics communication is definitely the future of data communication. The evolution of fiber optic communication has been driven by advancement in technology and increased demand for fiber optic communication. It is expected to continue into the future, with the development of new and more advanced communication technology. Below are some of the envisioned future trends in fiber optic communication.

\section{A. All Optical Communication Networks}

An all fiber optic communication is envisioned which will be completely in the optical domain, giving rise to an all 


\section{International Journal of Engineering Applied Sciences and Technology, 2020 \\ Vol. 4, Issue 10, ISSN No. 2455-2143, Pages 203-207 \\ Published Online February 2020 in IJEAST (http://www.ijeast.com)}

optical communication network. In such networks, all signals will be processed in the optical domain, without any form of electrical manipulation. Presently, processing and switching of signals take place in the electrical domain, optical signals must first be converted to electrical signal before they can be processed, and routed to their destination. After the processing and routing, the signals are then re-converted to optical signals, which are transmitted over long distances to their destination. This optical to electrical conversion, and vice versa, results in added latency on the network and thus is a limitation to achieving very high data rates.

Another benefit of all optical networks is that there will not be any need to replace the electronics when data rate increases, since all signal processing and routing occurs in the optical domain [9]. However, before this can become a reality, difficulties in optical routing, and wavelength switching has to be solved. Research is currently ongoing to find an effective solution to these difficulties.

\section{B. Multi-Terabit Optical Networks}

Dense Wave Division Multiplexing (DWDM) paves the way for multi-terabit transmission. The world-wide need for increased bandwidth availability has led to the interest in developing multi-terabit optical networks. Presently, four terabit networks using $40 \mathrm{~Gb} / \mathrm{s}$ data rate combined with 100 DWDM channels exists. Researchers are looking at achieving even higher bandwidth with $100 \mathrm{~Gb} / \mathrm{s}$. With the continuous reduction in the cost of fiber optic components, the availability of much greater bandwidth in the future is possible.

\section{Intelligent Optical Transmission Network}

Presently, traditional optical networks are not able to adapt to the rapid growth of online data services due to the unpredictability of dynamic allocation of bandwidth, traditional optical networks rely mainly on manual configuration of network connectivity, which is time consuming, and unable to fully adapt to the demands of the modern network. Intelligent optical network is a future trend in optical network development [2], and will have the following applications: traffic engineering, dynamic resource route allocation, special control protocols for network management, scalable signaling capabilities, bandwidth on demand, wavelength rental, wavelength wholesale, differentiated services for a variety of Quality of Service levels, and so on. It will take some time before the intelligent optical network can be applied to all levels of the network, it will first be applied in long-haul networks, and gradually be applied to the network edge [10].

\section{Ultra - Long Haul Optical Transmission}

In the area of ultra-long haul optical transmission, the limitations imposed due to imperfections in the transmission medium are subject for research. Cancellation of dispersion effect has prompted researchers to study the potential benefits of soliton propagation. More understanding of the interactions between the electromagnetic light wave and the transmission medium is necessary to proceed towards an infrastructure with the most favorable conditions for a light pulse to propagate [11].

\section{E. Improvements in Laser Technology}

Another future trend will be the extension of present semiconductor lasers to a wider variety of lasing wavelengths [12]. Shorter wavelength lasers with very high output powers are of interest in some high density optical applications. Presently, laser sources which are spectrally shaped through chirp managing to compensate for chromatic dispersion are available. Chirp managing means that the laser is controlled such that it undergoes a sudden change in its wavelength when firing a pulse, such that the chromatic dispersion experienced by the pulse is reduced. There is need to develop instruments to be used to characterize such lasers. Also, single mode tunable lasers are of great importance for future coherent optical systems. These tunable lasers lase in a single longitudinal mode that can be tuned to a range of different frequencies.

\section{F. $\quad$ Laser Neural Network Nodes}

The laser neural network is an effective option for the realization of optical network nodes. A dedicated hardware configuration working in the optical domain and the use of ultra-fast photonic sections is expected to further improve the capacity and speed of telecommunication networks [12]. As optical networks become more complex in the future, the use of optical laser neural nodes can be an effective solution.

\section{G. Polymer Optic Fibers}

Polymer optical fibers offer many benefits when compared to other data communication solutions such as copper cables, wireless communication systems, and glass fiber. In comparison with glass optical fibers, polymer optical fibers provide an easy and less expensive processing of optical signals, and are more flexible for plug interconnections [13]. The use of polymer optical fibers as the transmission media for aircrafts is presently under research by different Research and Development groups due to its benefits. The German Aerospace Center have concluded that "the use of Polymer Optical Fibers multimedia fibers appears to be possible for future aircraft applications [14]. Also, in the future, polymer optical fibers will likely displace copper cables for the last mile connection from the telecommunication company's last distribution box and the served end consumer [15]. The future Gigabit Polymer Optical Fiber standard will be based on TomlinsonHarashima Precoding, Multilevel PAM Modulation, and Multilevel Coset Coding Modulation.

\section{H. High-Altitude Platforms}

Presently, optical inter satellite links and orbit-to-ground links exists [16], the latter suffering from unfavorable weather conditions [17]. Current research explores optical communication to and from high altitude platforms. High altitude platforms are airships situated above the clouds at heights of 16 to $25 \mathrm{Km}$, where the unfavorable atmospheric 


\section{International Journal of Engineering Applied Sciences and Technology, 2020 \\ Vol. 4, Issue 10, ISSN No. 2455-2143, Pages 203-207 \\ Published Online February 2020 in IJEAST (http://www.ijeast.com)}

impact on a laser beam is less severe than directly above the ground [18]. As shown in figure 4, optical links between high- altitude platforms, satellites and ground stations are expected to serve as broadband back-haul communication channels, if a high-altitude platform functions as a data relay station.

\section{Improvementsin OpticalTransmitter/Receiver Technology}

In fiber optics communication, it is important to achieve high quality transmission even for optical signals with distorted waveform and low signal to noise ratio during transmission. Research is ongoing to develop optical transceivers adopting new and advanced modulation technology, with excellent chromatic dispersion and Optical Signal to Noise Ratio (OSNR) tolerance, which will be suitable for ultra-long haul communication systems. Also, better error correction codes, which are more efficient than the present $\mathrm{BCH}$ concatenated codes are envisioned to be available in the nearest future.

\section{Improvementin OpticalAmplification Technology}

Erbium Doped Fiber Amplifier (EDFA) is one of the critical technologies used in optical fiber communication systems. In the future, better technologies to enhance EDFA performance will be developed. In order to increase the gain bandwidth of EDFA, better gain equalization technology for high accuracy optical amplification will be developed. Also, in order to achieve a higher output power, and a lower noise figure, high power pumping lasers that possess excellent optical amplification characteristics with outputs of more than $+20 \mathrm{dBm}$, and very low noise figure are envisioned to exist in the nearest future.

\section{J. Advancement in Network Configuration of Optical Submarine Systems}

In order to improve the flexibility of network configuration in optical submarine communication systems, it is expected that the development of a technology for configuring the mesh network will be a step in the right direction. As shown in figure 5, while a ring network joins stations along a single ring, a mesh network connects stations directly. Presently, most large scale optical submarine systems adopt the ring configuration. By adopting the optical add/drop multiplexing technology that branches signals in the wavelength domain, it is possible to realize mesh network configuration that directly inter-connects the stations. Research is ongoing, and in the future such network configuration will be common.

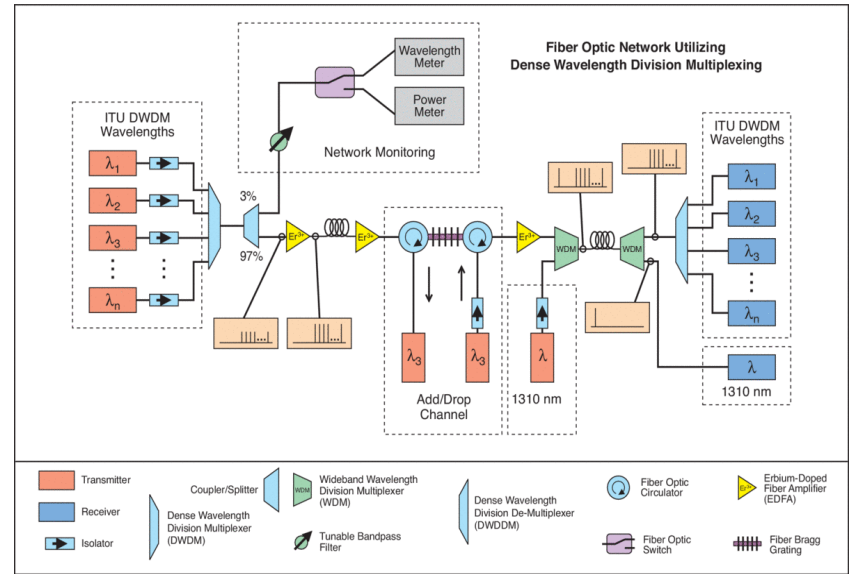

Fig.5. Optical Network Configurations [8]

\section{K. Improvement in WDM Technology}

Research is ongoing on how to extend the wavelength range over which wave division multiplexing systems can operate. Presently, the wavelength window ( $\mathrm{C}$ band) ranges from $1.53-1.57 \mu \mathrm{m}$. Dry fiber which has a low loss window promises an extension of the range to $1.30-1.65 \mu \mathrm{m}$. Also, developments in optical filtering technology for wave division multiplexing are envisioned in the future.

\section{Improvements in Glass Fiber Design and Component Miniaturization}

Presently, various impurities are added or removed from the glass fiber to change its light transmitting characteristics. The result is that the speed with which light passes along a glass fiber can be controlled, thus allowing for the production of customized glass fibers to meet the specific traffic engineering requirement of a given route. This trend is anticipated to continue in the future, in order to produce more reliable and effective glass fibers. Also, the miniaturization of optical fiber communication components is another trend that is most likely to continue in the future.

\section{CONCLUSION}

The fiber optics communications industry is an ever evolving one, the growth experienced by the industry has been enormous this past decade. There is still much work to be done to support the need for faster data rates, advanced switching techniques and more intelligent network architectures that can automatically change dynamically in response to traffic patterns and at the same time be cost efficient. The trend is expected to continue in the future as breakthroughs already attained in the laboratory will be extended to practical deployment thereby leading to a new generation in fiber optics communications.

\section{REFERENCES}

[1] M. Noshada, A. Rostami, "FWM minimization in WDM optical communication systems using the asymmetrical dispersion managed fibers", International Journal for Light and Electron Optics, 


\section{International Journal of Engineering Applied Sciences and Technology, 2020 \\ Vol. 4, Issue 10, ISSN No. 2455-2143, Pages 203-207 \\ Published Online February 2020 in IJEAST (http://www.ijeast.com)}

vol. 123, no. 9, pp. 758-760, 2012.

[2] X. Wang and K. Kitayama, "Analysis of beat noise in coherent and incoherent time-spreading OCDMA," IEEE/OSA Journal of Lightwave Technology ,vol. 22, no. 10, pp. 2226-2235, 2004.

[3] T. H. Shake, "Confident performance of encoded optical CDMA", IEEE/OSA Journal of Lightwave Technology, vol. 23, pp. 1652- 1663, 2005.

[4] Prachi Sharma et al, "A Review of the Development in the Field of Fiber Optic Communication Systems", International Journal of Emerging Technology and Advanced Engineering, Vol. 3, no. 5, pp. 113-119, 2013.

[5] G. Keiser, op cit, p 51

[6] Franz Fidler, Markus Knapek, Joachim Horwath, and Walter R.Leeb, "Optical Communications for High-Altitude Platforms", IEEE Journal of Selected Topics in Quantum Electronics, Vol. 16, no. 5, September/October 2010.

[7] T. Otani, K. Goto, H. Abe, M. Tanaka, H. Yamamoto, and H.Wakabayashi, Electron. Lett.31, 380, 1995.

[8] Ogata Takaaki, "Recent Status and Trends in Optical Submarine Cable Systems", NEC Technical Journal, Vol. 5 (1), pp. 4-7, 2010.

[9] Colin Yao, "The Future of Fiber Optic Communication", available at: www.streetdirectory.com, 2013.

[10] "Status of Optical Communication Technology and Future Trends", available at: www.qqread.net, 2013.

[11] Djan Kloe, Henrie Van Den Boom, "Trends in Electro-optical Communication Systems, Perspectives on Radio Astronomy: Technologies for Large Antenna Arrays, Proceedings of the Conference held at the ASTRON Institute in Dwingeloo on 12-14 April 1999. Edited by A. B. Smolders and M. P. Haarlem. Published by ASTRON. ISBN: 90-805434-2-X, 354 pages, 2000., p.2851999.

[12] Pamela L. Derry, Luis Figueroa, Chi Shain Hong, "Semi-Conductor Lasers", 1991.

[13] U.H.P. Fischer, M.Haupt and M.Janoic, "Optical Transmission Systems Using Polymeric Fibers", In Tech, available from: http://www.intechopen.com/books, 2011.

[14] Cherian, S., Spangenberg, H. and Caspary, R., "Vistas and Challenges for Polymer Optical Fiber in Commercial Aircraft, Proceedings of the 19th POF Conference, 2010.

[15] Koonen, A.M.J. et al, "POF Application in Home Systems and Local System", Proceedings of the 14th POF Conference, pp. 165-168, 2005.

[16] T. Jono, Y. Takayama, K. Shiratama, I. Mase, B. Demelenne, Z. Sodnik,A. Bird, M. Toyoshima, H. Kunimori, D. Giggenbach, N. Perlot,M. Knapek, and K. Arai, Overview of the inter-orbit and the orbit toground laser communication demonstration by OICETS, SPIE,vol. 6457, pp. 645702-1-645702-10, 2007.

[17] COST297. HAPCOS, "High Altitude Platforms for Communications and Other Services", Available Online at: http://www.hapcos.org, 2010.
[18] L. C. Andrews and R. L. Phillips, Laser Beam Propagation through Random Media, 2nd ed. Bellingham, WA: SPIE, 2005. 